\title{
Short-range order effect on resonance energy transfer in rigid solution
}

\author{
E.N. Bodunov ${ }^{\text {a }}$, M.N. Berberan-Santos ${ }^{\text {b,* }}$ \\ a Physics Department, Russian State Hydrometeorological University, St. Petersburg, 195196 Russia \\ ${ }^{\mathrm{b}}$ Centro de Química-Física Molecular, Instituto Superior Técnico, 1049-001 Lisboa, Portugal
}

Received 23 January 2004; accepted 23 February 2004

Available online 18 March 2004

\begin{abstract}
Resonance energy transfer by the Förster-Dexter mechanism in a rigid homogeneous medium is modeled using a hard-sphere fluid (HSF) radial distribution function. This distribution is more realistic than the commonly used uniform distribution with excluded volume (UDEV) function. For the dipole-dipole mechanism, both models yield essentially the same donor luminescence decay, except for small critical radii. For the exchange mechanism, however, the two models differ significantly. The HSF model displays a stronger "two-exponential" behavior. Also, to fit a given experimental decay, the UDEV model requires both a larger effective Bohr radius and a larger rate constant at collisional distance than the HSF model.
\end{abstract}

(c) 2004 Elsevier B.V. All rights reserved.

Keywords: Resonance energy transfer; Fluorescence; Luminescence; Liquid structure

\section{Introduction}

Current models of intermolecular quenching of luminescence (both fluorescence and phosphorescence) by nonradiative resonance energy transfer (RET) are largely based on the pioneering results of Förster [1] and Dexter [2], remarkable for their simplicity and accuracy [3-7]. Recent developments [6,8] have refined and helped to establish the limits of validity of these early approaches, but moderate quantitative improvements are achieved only at the cost of considerable computational work imposed by molecular details [8]. For most studies not involving molecular aggregates, the modeling of molecules as structureless particles seems to be a fairly accurate approximation, even for distances as short as a few angstrom $[9,10]$. In this work, calculations will be exclusively based on the basic Förster-Dexter theory, the purpose being to investigate the effect of short-range order on the luminescence decay of the donor.

\footnotetext{
${ }^{*}$ Corresponding author. Tel.: +35-121-841-9254; fax: +35-121-8464455.

E-mail address: berberan@ist.utl.pt (M.N. Berberan-Santos).
}

\section{General decay law}

In the modeling of RET between neutral molecules in a homogeneous medium, the donor and the acceptors surrounding it are usually assumed to be point-like and randomly distributed in space. In this case, the donoracceptor radial distribution function, $g(r)$, does not depend on the distance $r$ between the particles and is equal to unity for $0 \leqslant r<\infty$, i.e., the quenchers are uniformly and independently distributed around the excited molecule. The donor luminescence decay with quenching (normalized to unity at initial time) is more generally given by

$$
\begin{aligned}
I(t)= & \exp \left(-t / \tau_{0}\right) \exp \left(-4 \pi n_{\mathrm{q}}\right. \\
& \left.\times \int_{0}^{\infty}\left(1-\mathrm{e}^{-k(r) t}\right) g(r) r^{2} \mathrm{~d} r\right),
\end{aligned}
$$

where $\tau_{0}$ is intrinsic lifetime of the donor, $n_{\mathrm{q}}$ is the quencher concentration (number density), and $k(r)$ is the RET rate constant. This equation can be rewritten as

$I(t)=\exp \left(-t / \tau_{0}\right) \exp (-c H(t))$ 
where

$H(t)=4 \pi \int_{1}^{\infty}\left(1-\mathrm{e}^{-k(x) t}\right) g(x) x^{2} \mathrm{~d} x$,

and where $c=n_{\mathrm{q}} d^{3}, x=r / d$ and $d$ is the distance of closest approach, whenever $d>0$. In the derivation of Eq. (1), it is supposed that the concentration of donor molecules is very small, and that donor and acceptor molecules do not diffuse significantly during the donor lifetime. In the case of RET by the dipole-dipole mechanism [1]

$k(r)=\frac{1}{\tau_{0}}\left(\frac{R_{0}}{r}\right)^{6}$,

where $R_{0}$ is the critical or Förster radius, determined by the overlap of donor luminescence and acceptor absorption spectra, donor quantum yield, and refractive index of medium. The parameter $R_{0}$ takes values between 10 and $70 \AA$ [4-7].

For RET by the exchange mechanism [2],

$k(r)=k(0) \exp (-2 r / L)$,

where $k(0)$ is the quenching rate for the zero distance between donor and acceptor and $L$ is the so-called effective average Bohr radius. The exponential distance dependence of Eq. (5) results from the extent of spatial overlap of the electron clouds of excited molecule and quencher. The parameter $L$, initially introduced by Dexter [2] for RET between atoms by the exchange mechanism, originates from the simplified form of the electronic wave functions at large distances from the nuclei. In the molecular case, $L$ is best regarded as an empirical parameter [11]. For RET by the exchange mechanism, $L$ was reported to take values between 0.7 and $6 \AA[11,12]$, a typical value being ca. $1.5 \AA$.

Eqs. (4) and (5) are only approximate. Apart from the above mentioned deviations to the basic tenets of the Förster-Dexter formalism, the true rate constants are not only functions of relative distance but also of relative orientation. In the following, an isotropic interaction will be assumed for simplicity.

\section{Finite size of the molecules}

Molecules are also not point particles. The problem of RET between particles of finite size was first considered by Rikenglaz and Rozman $[13,14]$. The molecules were modeled as spheres, and the distance of closest approach $d$ was taken to be identical to the collisional radius. The radial distribution function used by these authors was the unit step function, i.e. a uniform distribution was assumed for distances larger than $d$,

$g(r)= \begin{cases}0 & \text { if } r<d \\ 1 & \text { if } r>d .\end{cases}$
This model will be called the uniform distribution with excluded volume, UDEV. Taking into account Eq. (6), Eq. (3) becomes

$H_{\mathrm{UDEV}}(t)=4 \pi \int_{1}^{\infty}\left(1-\mathrm{e}^{-k(x) t}\right) x^{2} \mathrm{~d} x$.

Note that the excluded volume is accounted in a proper way only for the donor-acceptor pair. Acceptors are still assumed to be independently distributed. This means that the model will not be adequate for very high acceptor concentrations [15-18].

Both the dipole-dipole [13] and exchange [14] mechanisms of RET were investigated in connection with this distribution. For the last case, it is preferable to rewrite the rate constant of RET, Eq. (5), as [11]

$$
\begin{aligned}
k(r) & =k_{0} \exp \left[-\frac{2(r-d)}{L}\right] \\
& =\frac{1}{\tau_{0}} \exp \left[\gamma-\frac{2(r / d-1)}{L / d}\right] .
\end{aligned}
$$

Now, $k_{0}$ is the quenching rate constant for the distance of closest approach, and $\gamma$ is a dimensionless parameter defined as $\gamma=\ln \left(k_{0} \tau_{0}\right)$. This parameter takes values from -3 to $20[11,13,14,19]$, depending on the system. Note that a low value of $\gamma$ can result either from a low absolute quenching effect (low $k_{0}$ ), or from a short intrinsic lifetime $\tau_{0}$. Likewise, a high value of $\gamma$ can result either from a high absolute quenching effect (high $k_{0}$ ), or from a long intrinsic lifetime $\tau_{0}$.

It is well known that the introduction of a distance of closest approach according to Eq. (6) leads to an exponential decay for short times $(k(d) t \ll 1$ in Eq. (7)). For long times, there are no noticeable differences between the decays with $d=0$ and $d>0$.

\section{The hard-sphere fluid}

In fact, the radial distribution function is a more complicated function of distance than a step function, Eq. (6). In liquids and molecular glasses, and even in the absence of a coulombic or van der Waals potential, a short-range order exists owing simply to the finite size of the molecules, and the radial distribution function has a damped oscillatory shape (see Fig. 1). If the density of fluid decreases, the amplitude of the oscillations decreases. The radial distribution function approaches the step function shape (UDEV model) only for a dilute gas. Several fluid models exist for the evaluation of $g(r)$. The simplest one is that of hard-spheres [20]. The hardsphere fluid (HSF) was namely used in [21,22] in the study of the rate constant of diffusion-controlled reactions, $k(t)$. It was shown that the time dependence of the reaction rate is the same as for the step function Eq. (6), with $k(t)=\alpha+\beta \sqrt{t}$ but the two parameters, $\alpha$ and $\beta$, have more realistic values. 


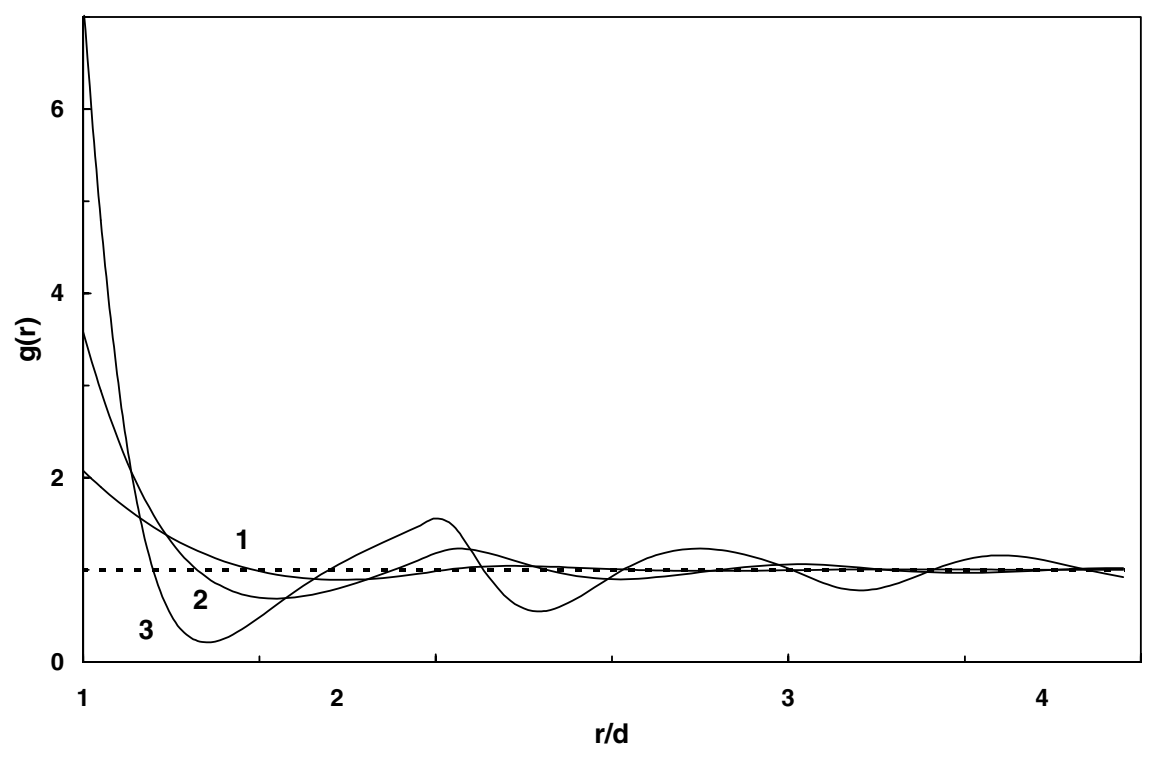

Fig. 1. Hard-sphere fluid (HSF) radial distribution function, $g(r)$, for fluid densities $\rho=0.5$ (1), 0.8 (2), and 1.1 (3).

To compute the luminescence decay Eq. (1), the appropriate radial distribution function $g(r)$ for the acceptors surrounding the donor must be used. This function was investigated for several models of liquid structure. We will use the hard-sphere fluid (HSF) model [20] owing to the following reasons. First, this model is the natural next step after modeling RET with the UDEV model. Second, the HSF has been investigated in detail: analytical solutions for $g(r)$ [23-28] and its Laplace transform [23] are known, and tables of $g(r)$ for different values of fluid density are available $[26,29,30]$. Third, the computed $g(r)$ for hard-spheres has an overall good agreement with simulation data, although some discrepancies are observed, especially at the highest densities [29,31].

In this paper, we will use the detailed tables given in [26]. The radial distribution function for several values of fluid dimensionless (or reduced) density $\rho$, where

$\rho=n d^{3}$,

$n$ and $d$ being the number density and the hard-sphere diameter of fluid molecules, respectively, is shown in Fig. 1.

The reduced density $\rho$ is connected to the packing density, $\eta$, which is the fraction of the volume occupied by the spheres, by

$\rho=\frac{6}{\pi} \eta$

hence for the most compact lattice packing possible in three-dimensional space (face-centered cubic packing, hexagonal close-packing) [32], where $\eta=\pi / 3 \sqrt{2} \cong 0.74$, one has $\rho=1.41$, while for a cubic lattice, where $\eta=\pi / 6 \cong 0.52, \rho=1.00$. For random close packing, $\eta \leqslant 0.64$ [33], hence $\rho \leqslant 1.22$.
There is a simple analytical solution for $g(r)$ at $r=d$ [25],

$g(d)=\frac{1+\eta / 2}{(1-\eta)^{2}}$.

To simplify the calculations, we will suppose that donor, acceptor, and solvent molecules are all spherical molecules with a common diameter, $d$. In this case, the radial distribution functions for the donor-acceptor pair in Eq. (3), and for a HSF coincide. For the calculations, $g(r)$ for $\rho=0.8$ and 1.1 is used. At these fluid densities, there is a marked difference between the step function Eq. (6) and $g(r)$ for a HSF: from Eq. (11) and [26], $g(d) \approx 3.58$ for $\rho=0.8$ and $g(d) \approx 7.16$ for $\rho=1.1$. Also, the hard-sphere solid and fluid phases are in thermodynamic equilibrium over a density interval from 0.943 to $1.04[30,34]$.

\section{Dipole-dipole mechanism}

For a uniform distribution of point-like molecules (UDP model, $g(r)=1$ for all $r$ ) and for the dipole-dipole interaction, it is well known that $H(t)$ in Eq. (3) takes the form

$H_{\mathrm{UDP}}(t)=\frac{4 \pi^{3 / 2}}{3}\left(\frac{R_{0}}{d}\right)^{3}\left(\frac{t}{\tau_{0}}\right)^{1 / 2}$.

Calculation of the donor luminescence decay according to the HSF model shows that a noticeable difference between the HSF and UDP models is observed only if $R_{0} / d<2$ (see Fig. 2 where $R_{0}=8 \AA, d=5 \AA$, and $\rho=1.1$ are used). This difference practically disappears if the value of $R_{0}$ used in Eq. (12) is but slightly 


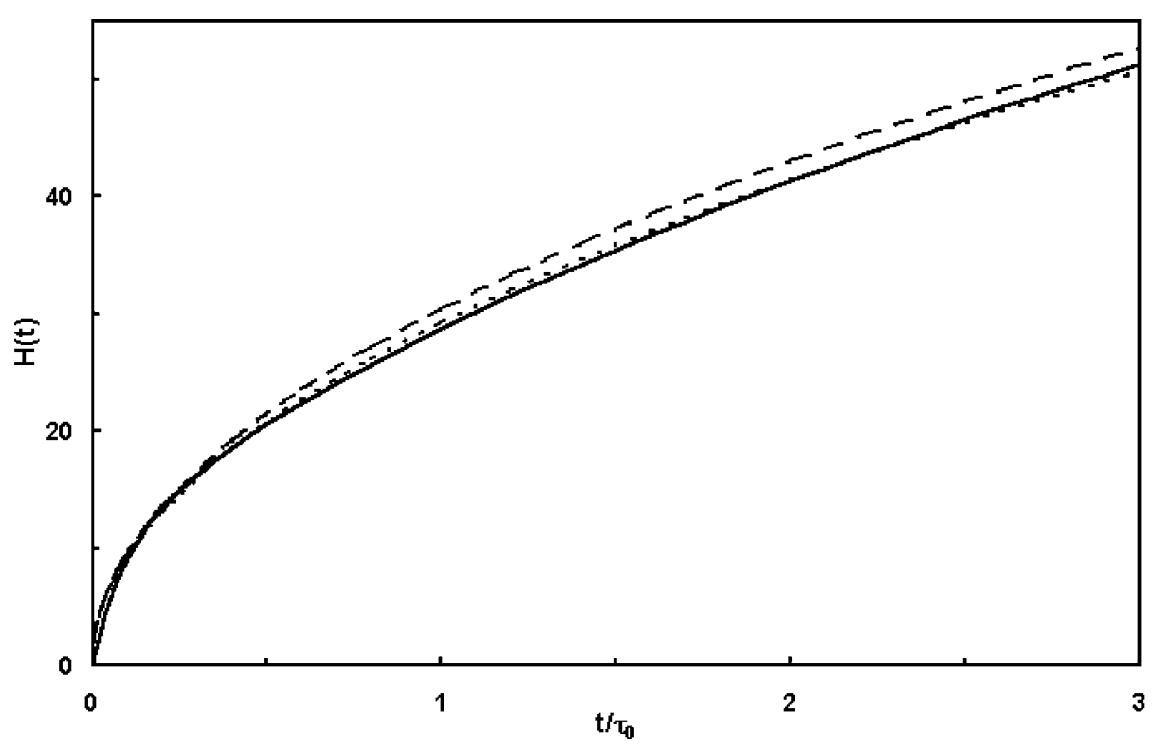

Fig. 2. Function $H(t)$ calculated for the dipole-dipole mechanism and for three different radial distribution functions. HSF model with density $\rho=1.1, d=5 \AA, R_{0}=8 \AA$ (solid line); UDP model, $R_{0}=8 \AA$ (dashed line) and $R_{0}=7.9 \AA$ (dotted line).

decreased (e.g. from 8 to $7.9 \AA$ in the case of the parameters used in Fig. 2).

The difference is more pronounced between the UDEV and the HSF models (Fig. 3). In this case, the Förster radius $R_{0}$ in Eq. (12) must be decreased from 8 to $7.7 \AA$ (other parameters being the same as in Fig. 3) to suppress the difference between the two decays in the time interval $0<t / \tau_{0}<3$.

The fact that the HSF model is closer to the UDP model than to the UDEV model results from the shape of the HSF radial distribution function. This function oscillates around 1 in a damped fashion, see Fig. 1, with values initially below unity $(g(r)=0$ for $0<r<d)$ and then above unity $(g(r)>1$ for $d \leqslant r<1.3 d$ ), which results in a nearly complete cancellation of effects.

\section{Exchange mechanism}

Recently [11], the UDEV model for fluorescence quenching with exponential distance dependence was studied in detail. It was shown that the parameters $L, d$ and $\gamma$ were correlated, and cannot be independently determined from a fluorescence decay. At most it is possible to determine $L$ and $\gamma$, while fixing $d$. Three different regimes were also identified: weak $(\gamma<2)$, in-

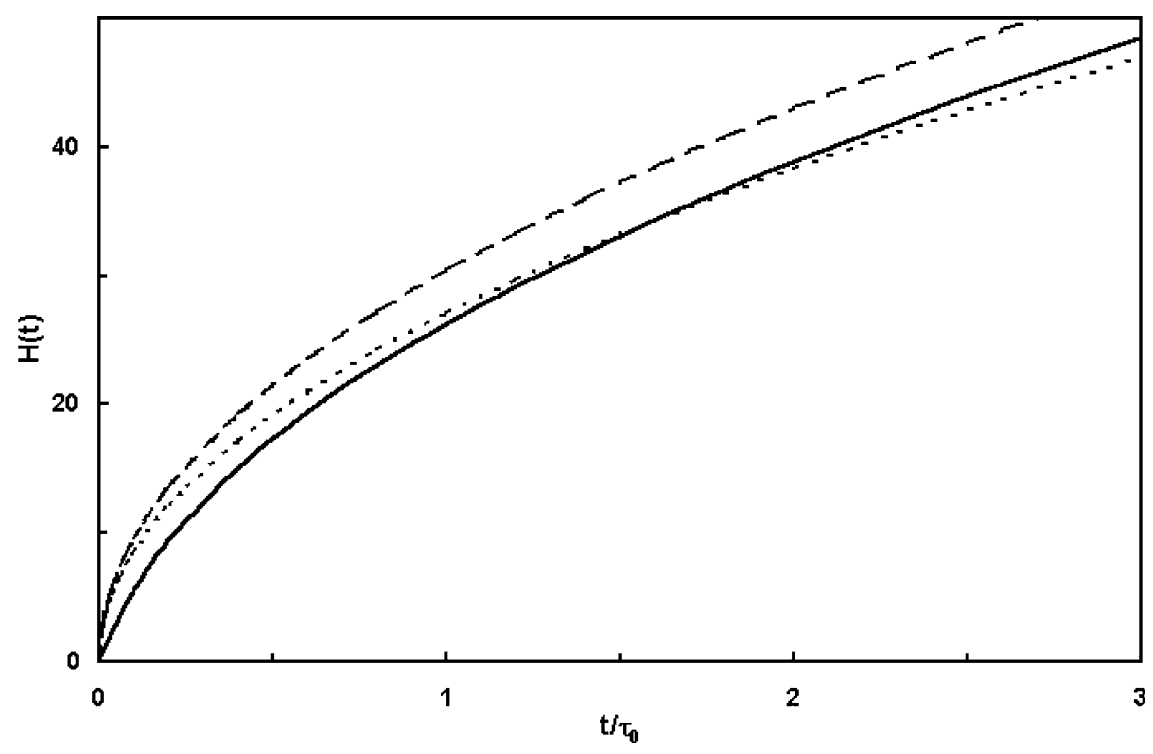

Fig. 3. Function $H(t)$ calculated for the dipole-dipole mechanism and for the HSF model with density $\rho=1.1, d=5 \AA$, $R_{0}=8 \AA$ (solid line); UDEV model, $d=5 \AA, R_{0}=8 \AA$ (dashed line) and $R_{0}=7.7 \AA$ (dotted line). 
termediate $(2<\gamma<5)$, and strong $(\gamma>5)$ quenching. Following [11], we will also consider such three cases. Namely, in our numerical calculation, we will use values $\gamma=-2,4$, and 10 and $L / d=0.2,0.5$, and 0.8 as typical experimental values $[4,11,19]$.

The HSF radial distribution function for $\rho=0.8$ and 1.1 is used. To simplify the comparison procedure (and in order not to take into account the effect of quencher concentration, $c$ ), only the function $H(t)$ is investigated. The function $H_{\mathrm{UDEV}}(t)$ will be called "theoretical", and the function $H_{\mathrm{HSF}}(t)$ "experimental".

In the weak quenching limit, $H(t)$ is almost a linear function of time. Assuming that the experimental precision is such that a linear expansion suffices, the experimental decay (HSF) becomes

$H(t)=H_{\mathrm{HSF}}(t) \approx 4 \pi \frac{t}{\tau_{0}} \mathrm{e}^{\gamma_{0}} \int_{1}^{\infty} \mathrm{e}^{-2(x-1) / b_{0}} g(x) x^{2} \mathrm{~d} x$,

and the theoretical one (UDEV),

$H(t)=4 \pi H_{\mathrm{UDEV}}(t) \approx \frac{t}{\tau_{0}} \mathrm{e}^{\gamma} \frac{1}{4} b\left(2+2 b+b^{2}\right)$.

As can be seen, $H_{\mathrm{HSF}}(t)$, can be fitted equally well by $H_{\mathrm{UDEV}}(t)$ for a continuous set of parameters $\gamma$ and $L / d$. For example, if $\rho=1.1, \gamma_{0}=-2$, and $L_{0} / d=0.2$, the theoretical curve fitting the experimental one can be obtained using sets of parameters from $\{\gamma=-0.49$, $L / d=0.18\}$ to $\{\gamma=-6.4, L / d=20\}$. Only when a quadratic term in time is introduced, does this correlation disappear [11].

In the intermediate case, the theoretical and experimental functions are no longer linear, and no satisfactory fit of the HSF decay with the UDEV model is possible in general. There is indeed a strong difference at short times in most cases (see Fig. 4). This difference depends on fluid concentration and decreases with the decrease of fluid density. The decay according to the HSF model has in general a marked two-exponential behavior, and the existence of a fast transient in the decay, not fully described by the UDEV model, is a sign of the existence of short-range order.

In the strong quenching limit $\left(\gamma_{0} \geqslant 10\right.$ and $L_{0} / d \geqslant 0.2$ or $\gamma_{0} \geqslant 4$ and $L_{0} / d \geqslant 0.5$ ), the experimental decays can be well fitted but the pairs of parameters $\left(\gamma_{0}, L_{0} / d\right)$ and $(\gamma, L / d)$ are different. This difference is nevertheless not large, and decreases with the increase of the values of the parameters. For example, if $\gamma_{0}=4, L_{0} / d=0.5$ in experimental decay, then $\gamma=4.3, L / d=0.49$ for the theoretical one. If $\gamma_{0}=10, L_{0} / d=0.8$ then $\gamma=10.09$, $L_{0} / d=0.8$. The precisions of the obtained values are\pm 0.05 for $\gamma$ and \pm 0.01 for $L / d$. Note that in the strong quenching limit, the obtained values are not dependent on the fluid concentration. They are quantitatively the same for $\rho=0.8$ and 1.1, although $g(d)$ for these fluid densities differ nearly by a factor of two, see Eq. (11). The situation approaches in this limit what was already observed for the dipole-dipole case in Section 3.

In [11], two molecular pairs were experimentally studied in rigid glasses at $77 \mathrm{~K}$ by picosecond singlephoton timing: $\mathrm{C}_{70}$-bromobenzene and phenanthreneiodide. In these systems the quenching of fluorescence occurs by the external heavy-atom effect, but the distance dependence is similar to that of the exchange mechanism. In the first system, the very different size of solvent $(7: 2(\mathrm{v} / \mathrm{v})$ methylcyclohexane-toluene) and one of the solute molecules $\left(\mathrm{C}_{70}\right)$ makes questionable the application of the HSF model as developed here, where solvent and solute are assumed to have identical size.

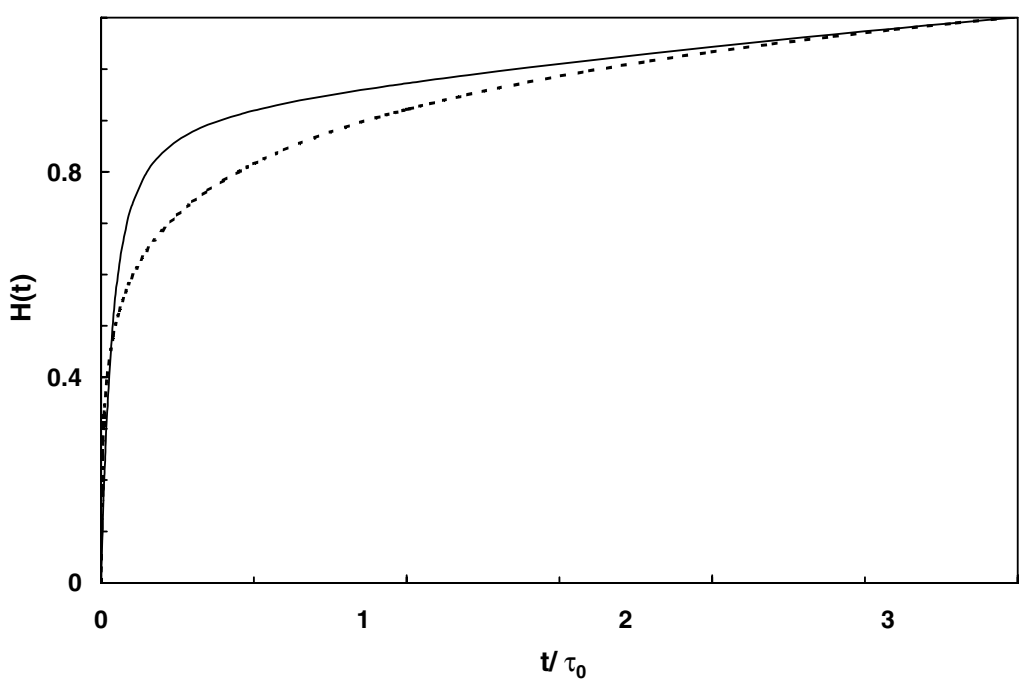

Fig. 4. Function $H(t)$ calculated for the exchange mechanism according to the HSF model $\left(\rho=1.1, d=5 \AA, \gamma_{0}=4, L_{0} / d=0.2\right.$, solid line) and to the UDEV model $(d=5 \AA, \gamma=7.9, L / d=0.13$, dotted line). Parameters $\gamma=7.9$ and $L / d=0.13$ of the dotted curve are chosen to bring together the two decays as much as possible. 
The second system, where all sizes are comparable, falls in the intermediate quenching case. Using $d=7.4 \AA$ and $\tau_{0}=64.4 \mathrm{~ns}$, the values $L=1.7 \AA$ and $k_{0}=0.12 \mathrm{~ns}^{-1}$ (that give $L / d=0.23$ and $\gamma=2.05$ ) were obtained [11] by fitting experimental fluorescence decays with the UDEV model. In this particular system, fits with the HSF model were also satisfactory. Different values for $\gamma$ and $L / d$ are obtained, and depend on the fluid density. For $\rho=0.8$, we obtain $L / d=0.17$ and $\gamma=1.23$ (or $L=1.3 \AA$ and $k_{0}=0.053 \mathrm{~ns}^{-1}$ ) for $t / \tau_{0}<3$. For a higher fluid density, $\rho=1.1, L / d=0.14$ and $\gamma=0.85$ (or $L=1.0 \AA$ and $k_{0}=0.036 \mathrm{~ns}^{-1}$ ) are obtained instead. Thus, for phenanthrene-iodide system, introduction of a more realistic radial distribution function (HSF model) allows to estimate parameters $L$ and $k_{0}$ as $1.0-1.3 \AA$ and $0.036-0.053 \mathrm{~ns}^{-1}$, respectively. These values are significantly smaller than those estimated with the UDEV model.

\section{Conclusions}

In this paper, the radial distribution function for the hard-sphere fluid model (HSF) was used to calculate the quenching of luminescence by dipole-dipole and exchange RET mechanisms. The HSF model takes into account the short-range order existent in fluids owing to the finite size of the molecules, which are modeled as rigid spheres.

For the dipole-dipole mechanism, the luminescence decay law obtained within the framework of this model does not practically differ from the usual Förster decay law for point-like particles, and from the decay law according to the UDEV model. The respective Förster radii differ only slightly.

For the short-ranged exchange mechanism, the decay laws obtained with the use of hard-sphere fluid radial distribution function and with the step function are practically identical if the quenching is strong, but the two sets of parameters $\gamma$ and $L / d$ are different. The parameter $L / d$ is smaller for the HSF model because the radial distribution function at the distance of closest approach is noticeably larger than unity. In the very weak quenching limit, parameters $\gamma$ and $L / d$ are correlated and cannot in principle be independently determined from luminescence decays. In the intermediate case, the luminescence decay laws usually have quite different time dependences for the uniform distribution with excluded volume and HSF radial distribution functions, but in some cases can be made to agree.

\section{Acknowledgements}

This work was carried out within project POCTI 34836/FIS/2000 from Fundação para a Ciência e a Tecnologia, FCT (Portugal).

\section{References}

[1] Th. Förster, Ann. Phys. 2 (1948) 55.

[2] D.L. Dexter, J. Chem. Phys. 21 (1953) 836.

[3] M.N. Berberan-Santos, Chem. Phys. Lett. 196 (1992) 220.

[4] V.L. Ermolaev, E.N. Bodunov, E.B. Sveshnikova, T.A. Shakhverdov, Radiationless Energy Transfer of Electronic Excitation, Nauka, Leningrad, 1977.

[5] M.D. Galanin, Luminescence of Molecules and Crystals, CISP, Cambridge, 1996.

[6] D.L. Andrews, A.A. Demidov (Eds.), Resonance Energy Transfer, Wiley, Chichester, 1999.

[7] B. Valeur, Molecular Fluorescence, Wiley-VCH, Weinheim, 2002.

[8] G.D. Scholes, Annu. Rev. Phys. Chem. 54 (2003) 57.

[9] M.N. Berberan-Santos, P. Choppinet, A. Fedorov, L. Jullien, B. Valeur, J. Am. Chem. Soc. 121 (1999) 2526.

[10] M.N. Berberan-Santos, P. Choppinet, A. Fedorov, L. Jullien, B. Valeur, J. Am. Chem. Soc. 122 (2000) 11876.

[11] M. Rae, A. Fedorov, M.N. Berberan-Santos, J. Chem. Phys. 119 (2003) 2223.

[12] A. Brown, F. Wilkinson, J. Chem. Soc., Faraday Trans. 2 (75) (1979) 880.

[13] M.M. Rikenglaz, I.M. Rozman, Opt. Spectrosc. 36 (1974) 100.

[14] M.M. Rikenglaz, I.M. Rozman, Opt. Spectrosc. 36 (1974) 106.

[15] S.I. Golubov, Y.V. Konobeev, Sov. Phys. Solid State 13 (1972) 2679.

[16] V.P. Sakun, Sov. Phys. Solid State 14 (1973) 1906.

[17] A. Blumen, J. Manz, J. Chem. Phys. 71 (1979) 4694.

[18] A. Blumen, J. Chem. Phys. 72 (1980) 2632.

[19] M. Inokuti, F. Hirayama, J. Chem. Phys. 43 (1965) 1978.

[20] J.K. Percus, G.J. Yevick, Phys. Rev. 110 (1958) 1.

[21] J.C. André, F. Baros, M. Bouchy, Chem. Phys. 103 (1986) 391

[22] F. Baros, J.C. André, React. Kinetics Catal. Lett. 36 (1988) 1.

[23] M.S. Wertheim, Phys. Rev. Lett. 10 (1963) 321.

[24] E. Thiele, J. Chem. Phys. 39 (1963) 474.

[25] J.L. Lebowitz, Phys. Rev. A 133 (1964) 895.

[26] G.J. Throop, R.J. Bearman, J. Chem. Phys. 42 (1965) 2408.

[27] W.R. Smith, D. Henderson, Mol. Phys. 19 (1970) 411

[28] S.B. Yuste, A. Santos, Phys. Rev. A 43 (1991) 5418.

[29] F.H. Ree, R.N. Keeler, S.L. McCarthy, J. Chem. Phys. 44 (1966) 3407.

[30] F.H. Ree, Y.-T. Lee, T. Ree, J. Chem. Phys. 55 (1971) 234.

[31] A. Barker, D. Henderson, Rev. Mod. Phys. 48 (1976) 587.

[32] J.H. Conway, N.J.A. Sloane, Sphere Packings, Lattices, and Groups, third ed., Springer-Verlag, New York, 1999.

[33] S. Torquato, T.M. Truskett, P.G. Debenedetti, Phys. Rev. Lett. 84 (2000) 2064.

[34] W.G. Hoover, F.H. Ree, J. Chem. Phys. 49 (1968) 3609. 